\title{
HIGHER JET EVALUATION TRANSVERSALITY OF $J$-HOLOMORPHIC CURVES
}

\author{
YONG-GEUN OH
}

\begin{abstract}
In this paper, we establish general stratawise higher jet evaluation transversality of $J$-holomorphic curves for a generic choice of almost complex structures $J$ (tame to a given symplectic manifold $(M, \omega)$ ).

Using this transversality result, we prove that there exists a subset $\mathcal{J}_{\omega}^{\text {ram }} \subset \mathcal{J}_{\omega}$ of second category such that for every $J \in \mathcal{J}_{\omega}^{\text {ram }}$, the dimension of the moduli space of (somewhere injective) $J$-holomorphic curves with a given ramification profile goes down by $2 n$ or $2(n-1)$ depending on whether the ramification degree goes up by one or a new ramification point is created.

We also derive that for each $J \in \mathcal{J}_{\omega}^{\text {ram }}$ there are only a finite number of ramification profiles of $J$-holomorphic curves in a given homology class $\beta \in H_{2}(M ; \mathbb{Z})$ and provide an explicit upper bound on the number of ramification profiles in terms of $c_{1}(\beta)$ and the genus $g$ of the domain surface.
\end{abstract}

\section{Contents}

1. Introduction

2. Jet evaluation map and holomorphic jets 346

3. Fredholm framework 349

4. Higher jet evaluation transversality 351

4.1. Statement 351

4.2. Proof 352

5. Removal of singularity: Proof of Lemma $4.7 \quad 359$

6. Stratawise transversality and finiteness of ramification profiles 362

References 365

Received October 26, 2009.

2010 Mathematics Subject Classification. Primary 53D35; 14H10.

Key words and phrases. higher jet evaluation transversality, holomorphic jets, ramification profiles, distributions with points support.

Partially supported by the NSF grant \#DMS 0503954. 


\section{Introduction}

Let $(M, \omega)$ be a symplectic manifold of dimension $2 n$. Denote by $J$ an almost complex structure tame to $\omega$ and by $\mathcal{J}_{\omega}$ the set of tame almost complex structures.

Let $\Sigma$ be an oriented compact surface without boundary of genus $g$ and $(j, u)$ be a pair of a complex structure $j$ on $\Sigma$ and a map $u: \Sigma \rightarrow M$. We say that $(j, u)$ is a $J$-holomorphic if it satisfies $J \circ d u=d u \circ j$. We denote the standard moduli spaces of $J$-holomorphic maps $(j, u)$ from $\Sigma$ to $M$ in class $[u]=\beta$ by $\widetilde{\mathcal{M}}_{g}(M, J ; \beta)$ and consider its quotient $\mathcal{M}_{g}(M, J ; \beta)=\widetilde{\mathcal{M}}_{g}(M, J ; \beta) / \operatorname{Aut}(\Sigma)$.

The main purpose of the present paper is to establish higher jet evaluation transversality whose precise formulation we refer to Section 4.

From this higher jet evaluation transversality, we derive stratawise transversality of ramification divisors whose statement is now in order.

Definition 1.1. The ramification degree of the map $u$ at a point $z \in \Sigma$ is defined to be the unique integer $k \in \mathbb{N}$ such that

$$
j^{k} u(z)=0, \quad \text { but } j^{k+1} u(z) \neq 0,
$$

where $j^{k} u(z)$ is the $k$-jet of the map $u$ at $z$. If there is no such $k$, we say $u$ has an infinite ramification degree. We say that any immersed point has ramification degree 0.

Basic results from [6], [10] on the structure of singularities of $J$-holomorphic map $(j, u)$ state that there are only finitely many critical points and that each critical point has a finite ramification degree. This motivates us to consider the set of pairs

$$
(k ; \vec{n}), \quad k \in \mathbb{Z}_{\geq 0} \text { and } \vec{n} \in \mathbb{N}^{k} .
$$

For each given $k$ distinct points $z=\left\{z_{1}, \ldots, z_{k}\right\}$, we consider the decoration of positive integers $n_{i}$ assigned at $z_{i}$ 's. We denote $\vec{n}=\left\{n_{1}, \ldots, n_{k}\right\}$ and $K=$ $\{1, \ldots, k\}$. For given $k \leq k^{\prime}$ and $K^{\prime}=\left\{1, \ldots, k^{\prime}\right\}$ we decompose

$$
K^{\prime}=K \cup\left(K^{\prime} \backslash K\right) \text {. }
$$

Definition 1.2. We say $\left(k^{\prime} ; \vec{n}^{\prime}\right)<(k ; \vec{n})$ if

$$
k \leq k^{\prime} \text { and } \quad n_{i} \leq n_{i}^{\prime} \text { for all } i \in K \subset K^{\prime} .
$$

We define the corresponding moduli space of $J$-holomorphic maps with prescribed ramifications at $k$ marked points

$$
\widetilde{\mathcal{M}}_{g, k}(M, J ; \beta, \vec{n})=\left\{((j, u), z) \mid \bar{\partial}_{(j, J)} u=0, \operatorname{deg}_{z_{i}}(u) \geq n_{i}\right\}
$$

and

$$
\mathcal{M}_{g, k}(M, J ; \beta, \vec{n})=\widetilde{\mathcal{M}}_{g, k}(M, J ; \beta, \vec{n}) / \operatorname{Aut}(\Sigma) .
$$

We emphasize, though, that an element $u$ from this moduli space could have other ramification points unmarked. 
Notation: Throughout the paper, we will abuse our notation and always denote by $\mathcal{M}_{g, k}(M, J ; \beta)$ the open subset consisting of somewhere injective $J$ holomorphic curves $(j, u)$ in the standard smooth moduli space which is usually denoted by the notation $\mathcal{M}_{g, k}(M, J ; \beta)$ itself. Similar remarks will apply to all other moduli spaces.

The following is one of the main theorems we prove in the present paper.

Theorem 1.3. There exists a subset $\mathcal{J}_{\omega}^{\text {ram }} \subset \mathcal{J}_{\omega}$ such that for any $J \in \mathcal{J}_{\omega}^{\text {ram }}$ the moduli space $\widetilde{\mathcal{M}}_{g, k}(M, J ; \beta ; \vec{n})$ is a smooth manifold of dimension

$$
\operatorname{dim} \widetilde{\mathcal{M}}_{g, k}(M, J ; \beta)-\sum_{i=1}^{k} 2 n n_{i}
$$

for all $\beta \in H_{2}(M)$ and $g \in \mathbb{Z}_{\geq 0}$.

Recall the standard dimension formula for

$$
\operatorname{dim} \widetilde{\mathcal{M}}_{g, k}(M, J ; \beta)= \begin{cases}2\left(c_{1}(M, \omega)(\beta)+(n-3)(1-g)\right)+2 k & \text { for } g \geq 2 \\ 2\left(c_{1}(M, \omega)(\beta)+1\right)+2 k & \text { for } g=1 \\ 2\left(c_{1}(M, \omega)(\beta)+n\right)+2 k & \text { for } g=0\end{cases}
$$

for the maps $u$ with $[u]=\beta \in H_{2}(\mathbb{Z})$.

Our proof of this theorem relies on a new Fredholm set-up we establish in this paper using the notion of holomorphic jet bundles. Using this Fredholm work and some judicious usage of a structure theorem of distributions with point support (see [5] for example), we prove a higher jet evaluation transversality which uses an extension of the scheme of the 1-jet transversality proof employed by Zhu and the present author in [9]. An important point used in our proof is the fact that the holomorphic jet bundles are canonically associated to the pair of a Riemann surface $(\Sigma, j)$ and an almost complex manifold $(M, J)$ in the 'off-shell' level, i.e., on the space of smooth maps, not just on the moduli space of $J$-holomorphic maps.

A priori, $\widetilde{\mathcal{M}}_{g, k}(M, J ; \beta ; \vec{n})$ 's are abstract manifolds residing independently from one another. The following theorem relates them when the corresponding ramification orders are right next to each other.

We have two kinds of immediate predecessors $\left(k^{\prime} ; \vec{n}^{\prime}\right)$ to $(k ; \vec{n})$ :

(a) $\left(k^{\prime} ; \vec{n}^{\prime}\right)=\left(k ; \vec{n}+\vec{e}_{i}\right)$ for some $1 \leq i \leq k$ where we denote by $\vec{n}+\vec{e}_{i}$ the decoration

$$
\left(n_{1}, \ldots, n_{i}+1, \ldots, n_{k}\right) .
$$

(b) $\left(k^{\prime} ; \vec{n}^{\prime}\right)=\left(k+1, \vec{n} \cup\left\{n_{k+1}\right\}\right)$ with $n_{k+1}=1$.

Theorem 1.4. For $J \in \mathcal{J}_{\omega}^{\text {ram }}$ and $\beta \in H_{2}(M)$ and $g \in \mathbb{Z}_{>0}$, the following holds: 
(1) For the type (a) of the immediate predecessor of $\left(k^{\prime} ; \vec{n}^{\prime}\right)=\left(k, \vec{n}+\vec{e}_{i}\right)$ for some $i=1, \ldots, k, \mathcal{M}_{g, k}\left(J ; \beta, \vec{n}+\vec{e}_{i}\right)$ is a smooth submanifold of $\mathcal{M}_{g, k}(J ; \beta, \vec{n})$ with its dimension $2 n$ smaller,

(2) For the type (b), the image of the forgetful map $\mathcal{M}_{g, k+1}(J ; \beta, \vec{n}+$ $\left.\vec{e}_{k+1}\right) \rightarrow \mathcal{M}_{g, k}(J ; \beta, \vec{n})$ induces an embedding of codimension $2(n-1)$.

It has been a folklore that "for a generic choice of $J$, the dimension of the moduli space of a given ramification profile goes down when either the ramification order goes up or a new ramification point is created". However it has not been clear what the precise statement of this folklore would really be. The above theorem provides a precise form of this folklore. The main stumbling block to make this folklore into a precise theorem has been what kind of moduli spaces one should look at to obtain the kind of anticipated dimension cutting-down statement hold. It took the author some time to find out which moduli space is the correct one with respect to which the necessary Fredholm framework can be carried out. Only after the work [9] which concerns the 1-jet evaluation transversality, the answer became clear to the author. This has led the author to the Fredholm setting used in the present paper.

Next for each given non-constant $J$-holomorphic map $(j, u)$, we associate to it the ramification profile:

Definition 1.5. A ramification profile, denoted by $\operatorname{ram}(u)$, of $(j, u)$ is a pair $(N ; \lambda)$ with $N \in \mathbb{Z}_{\geq 0}$ and a partition $\lambda=\left(\lambda_{1}, \ldots, \lambda_{L}\right) \vdash N$ of $N$ such that

$$
\begin{gathered}
\text { Crit } u=\left\{z_{1}, \ldots, z_{k}\right\} \subset \Sigma, \quad k=\sum_{\ell=1}^{L} a_{\ell}, \\
\operatorname{deg}_{z_{i}}(u)=d_{\ell} \quad \text { whenever } i \in \lambda_{\ell}, \quad l \leq \ell \leq L,
\end{gathered}
$$

where $L=|\lambda|$ is the length of the partition and $\lambda=\left(d_{1}^{a_{1}} \cdots d_{L}^{a_{L}}\right)$ to denote the partition that has $a_{j}$ points of $z_{i}$ that has the same degree $d_{\ell}$. We call $N$ the total ramification degree of $(j, u)$.

We note that

$$
N=\sum_{i=1}^{L} d_{i} a_{i}
$$

when $\lambda=\left(d_{1}^{a_{1}} \cdots d_{L}^{a_{L}}\right) \vdash N$. We denote by

$$
\widetilde{\mathcal{M}}_{g}^{(N ; \lambda)}(M, J ; \beta)
$$

the moduli space of $J$-holomorphic curves with ramification profile $(N ; \lambda)$. We study the cardinality of ramification profiles of (unmarked) $J$-holomorphic curves for a given genus $g$ and homology class $\beta \in H_{2}(M, \mathbb{Z})$. We have the obvious decomposition of the moduli space of unmarked $J$-holomorphic curves

$$
\widetilde{\mathcal{M}}_{g}(M, J ; \beta)=\bigcup_{N \in \mathbb{Z} \geq 0} \bigcup_{\lambda \in P(N)} \widetilde{\mathcal{M}}_{g}^{(N ; \lambda)}(M, J ; \beta) .
$$


The relation between $\widetilde{\mathcal{M}}_{g}^{(N ; \lambda)}(M, J ; \beta)$ and $\widetilde{\mathcal{M}}_{g, k}(M, J ; \beta ; \vec{n})$ is as follows. For a given partition $\lambda=\left(d_{1}^{a_{1}} \cdots d_{L}^{a_{L}}\right)$ with $L=|\lambda|$, we consider the moduli space $\mathcal{M}_{g, k}(M, J ; \beta ; \vec{n})$ with $\vec{n}=\left(n_{1}, \ldots, n_{L}\right)$. We consider the forgetful map

$$
\operatorname{forget}_{(k ; \vec{n})}: \widetilde{\mathcal{M}}_{g, k}(M, J ; \beta ; \vec{n}) \rightarrow \widetilde{\mathcal{M}}_{g}(M, J ; \beta) .
$$

Conversely when $(k ; \vec{n})$ is given, we can associate a unique partition $(N ; \lambda)$ given by

$$
N=\sum_{i=1}^{k} n_{i}, \lambda=\left(n_{i_{1}}^{a_{1}} \cdots n_{i_{L}}^{a_{L}}\right), \quad k=\sum_{\ell=1}^{L} a_{\ell}
$$

for $n_{1}=\cdots=n_{i_{1}}>n_{i_{1}+1}=\cdots=n_{i_{2}}>\cdots>n_{i_{L}}$. Then we have

$$
\begin{aligned}
\widetilde{\mathcal{M}}_{g}^{(N ; \lambda)}(M, J ; \beta)=\operatorname{forget}_{(k ; \vec{n})}\left(\widetilde{\mathcal{M}}_{g, k}(M, J ; \beta ; \vec{n})\right) \\
\qquad \bigcup_{\left(k^{\prime} ; \vec{n}^{\prime}\right)<(k ; \vec{n})} \mathfrak{f o r g e t}_{\left(k^{\prime} ; \vec{n}^{\prime}\right)}\left(\widetilde{\mathcal{M}}_{g, k^{\prime}}\left(M, J ; \beta ; \vec{n}^{\prime}\right)\right) .
\end{aligned}
$$

We note that an element from $\widetilde{\mathcal{M}}_{g}^{(N ; \lambda)}(M, J ; \beta)$ will have their ramification points and ramification orders exactly the same as prescribed. A priori, the union (1.3) could be an infinite union.

The following theorem says that this will be a finite union for a generic choice of $J$.

Theorem 1.6. Let $\beta \in H_{2}(M, \mathbb{Z})$ and $g$ be given. Then for any $J \in \mathcal{J}_{\omega}^{\text {ram }}$, the number of types of ramification profiles of $\mathcal{M}_{g}(M, J ; \beta)$ is not bigger than

$$
P\left(c_{1}(\beta)+(3-n)(g-1)\right),
$$

that is, the number of partitions of the integer $c_{1}(\beta)+(3-n)(g-1)$, when $c_{1}(\beta)+(3-n)(g-1) \geq 0$.

We note that if $c_{1}(\beta)+(3-n)(g-1)-n<0$, then the corresponding moduli space will be empty. And we also emphasize that each stratum of the union (1.3) could have singularities as a subset of $\widetilde{\mathcal{M}}(M, J ; \beta)$.

The statements in the above theorems are somewhat reminiscent of the Noetherian property of holomorphic maps in projective algebraic varieties. We find it curious that this kind of finite statements hold in two opposite ends of the category and wonder if there is any universal phenomenon in that direction.

The Fredholm framework and the scheme of the relevant evaluation transversality proof that we employ in the present paper are the higher jet analogs to the ones used for the 1 jet evaluation transversality studied in [9]. A similar higher jet analysis is also carried out in [8] in relation to the compactification of the moduli space of smooth holomorphic sections of the (singular) Lefschetz Hamiltonian fibrations.

We thank Zhu for having many enlightening discussions during the collaboration of the work [9] and other projects. We thank the referee pointing out 
Barraud's paper [1] in which a similar definition of higher jet spaces and evaluation maps for $J$-holomorphic curves is given: Barraud provided a sufficient condition for $J$ to be generic in a certain sense restricted to dimension 4 but did not prove the existence of such generic $J$. We also thank the referee for suggesting to make the definition of ramification profiles precise which led us to the definition of ramification profiles in this version.

\section{Jet evaluation map and holomorphic jets}

In this section, we study a smooth map $u: \Sigma \rightarrow M$ whose first $k$ derivatives vanish $z \in \Sigma$, i.e.,

$$
j^{k} u(z)=0
$$

where $j^{k} u(z)$ is the $k$-jet of the map $u$. For such a map, we would like to say that the $(k+1)$-th derivative of $(j, J)$-holomorphic map $u$ at $z$ induces a canonical linear map from $T_{z} \Sigma \rightarrow H_{z, u(z)}^{(k+1,0)} \cong T_{u(z)} M$, where $H_{z, u(z)}^{(k+1,0)}$ is the set of 'holomorphic part' of the $(k+1)$-jet space. We will make this statement precise in the rest of the section.

For this purpose, we recall the definition of $k$-jet bundle and the $k$-jet $j^{k} u(z)$ at $z \in \Sigma$ (See [3] for a nice exposition on the jet bundle). The $k$-jet bundle $J^{k}(\Sigma, M) \rightarrow \Sigma \times M$ is the vector bundle whose fiber at $(z, x)$ is given by

$$
J_{(z, x)}^{k}(\Sigma, M)=P^{k}\left(T_{z} \Sigma, T_{x} M\right),
$$

where $P^{k}\left(T_{z} \Sigma, T_{x} M\right)$ is the set of polynomial maps from $T_{z} \Sigma$ to $T_{x} M$ of degree $\leq k$, or

$$
P^{k}\left(T_{z} \Sigma, T_{x} M\right)=\prod_{\ell=0}^{k} \operatorname{Sym}^{\ell}\left(T_{z} \Sigma, T_{x} M\right),
$$

where $\operatorname{Sym}^{\ell}\left(T_{z} \Sigma, T_{x} M\right)$ is the vector space of symmetric $\ell$-linear maps from $T_{z} \Sigma \rightarrow T_{x} M$.

We have a natural sequence of bundles over $\Sigma \times M$

$$
J^{0}(\Sigma, M) \leftarrow J^{1}(\Sigma, M) \leftarrow \cdots \leftarrow J^{k}(\Sigma, M) \leftarrow
$$

and

$$
J_{x}^{0}(\Sigma, M) \leftarrow J_{x}^{1}(\Sigma, M) \leftarrow \cdots \leftarrow J_{x}^{k}(\Sigma, M) \leftarrow .
$$

Here the map $\pi: J^{k+1}(\Sigma, M) \rightarrow J^{k}(\Sigma, M)$ is defined by the 'truncation' of polynomial map $P$ to the terms of order $\leq k$. We denote by $P \leq k$ the truncation of $P$ thereto. Then we have

$$
\pi(z, x ; P)=\left(z, x ; P^{\leq k}\right) .
$$

Now we consider the space

$$
\mathcal{F}_{1}(\Sigma, M)=\{(u, z) \mid u: \Sigma \rightarrow M, z \in \Sigma\} .
$$

We have the natural $k$-jet evaluation map

$$
j^{k}: \mathcal{F}_{1}(\Sigma, M) \rightarrow J^{k}(\Sigma, M) ; j^{k}(u, z)=j_{z}^{k} u
$$


and for a fixed point $z \in \Sigma$

$$
j_{z}^{k}: \mathcal{F}(\Sigma, M) \rightarrow J_{z,(\cdot)(z)}^{k}(\Sigma, M) ; j_{z}^{k}(u)=j_{z}^{k} u .
$$

We have a short exact sequence

$$
0 \rightarrow \operatorname{ker} \pi \rightarrow J^{k+1}(\Sigma, M) \stackrel{\pi}{\rightarrow} J^{k}(\Sigma, M) \rightarrow 0
$$

of vector bundles over $\Sigma \times M$. By construction, we have

$$
\pi \circ j^{k+1}=j^{k} \text {. }
$$

Now we equip $\Sigma$ and $M$ with almost complex structures $j$ and $J$ respectively. The almost complex structures $j$ on $\Sigma$ and $J$ on $M$ naturally split off the direct summands of $\operatorname{Sym}^{k}(\Sigma, M)$ such as

$$
\operatorname{Sym}^{k}(\Sigma, M)=\operatorname{Sym}_{\left(j_{z}, J_{x}\right)}^{(k, 0)}(\Sigma, M) \oplus \operatorname{Sym}_{\left(j_{z}, J_{x}\right)}^{(0, k)}(\Sigma, M) \oplus \text { "mixed parts". }
$$

Definition 2.1. We call an element $L \in \operatorname{Sym}^{k}\left(T_{z} \Sigma, T_{x} M\right)$ holomorphic at $(z, x)$ (relative to $(j, J))$ if $L$ lies in $H_{z, x}^{(k, 0)}(\Sigma, M):=\operatorname{Sym}_{\left(j_{z}, J_{x}\right)}^{(k, 0)}(\Sigma, M)$.

We note that the vector space $\operatorname{Sym}_{\left(j_{z}, J_{x}\right)}^{(k ; 0)}(\Sigma, M)$ has the same dimension as $T_{x} M$ for all $k$, which is nothing but $2 n$.

We denote

$$
J_{h o l,(z, x)}^{k}(\Sigma, M)=\bigoplus_{\ell=0}^{k} H_{(z, x)}^{(\ell, 0)}(\Sigma, M)
$$

and form the union

$$
J_{h o l}^{k}(\Sigma, M)=\bigcup_{(z, x) \in \Sigma \times M} J_{h o l,(z, x)}^{k}(\Sigma, M)
$$

which we call the holomorphic jet bundle of $\Sigma \times M$ relative to $(j, J)$.

Then we have the natural projection

$$
\pi_{(j, J)}^{h o l}: J^{k}(\Sigma, M) \rightarrow J_{h o l}^{k}(\Sigma, M)
$$

and the inclusion

$$
i_{(j, J)}^{h o l}: J_{h o l}^{k}(\Sigma, M) \rightarrow J^{k}(\Sigma, M) .
$$

Now consider a $C^{k+1}$-map $u: \Sigma \rightarrow M$. We define the $k$-th holomorphic jet of $u: \Sigma \rightarrow M$ by

$$
j_{h o l}^{k} u(z):=\pi_{(j, J)}^{h o l}\left(j^{k} u(z)\right)=\bigoplus_{\ell=0}^{k} \pi_{j, J}^{h o l}\left(d^{\ell} u(z)\right)
$$

and

$$
\sigma^{k}(J,(j, u), z):=\pi_{(j, J)}^{h o l}\left(d^{k} u(z)\right)
$$


Definition 2.2. Let $u: \Sigma \rightarrow M$ be a smooth map satisfying

$$
j^{k} u(z)=0 \quad \text { but } j^{k+1} u(z) \neq 0 .
$$

We say that $u$ has ramification degree $k$ and $j^{k+1} u(z)$ the principal jet of $u$. The holomorphic ramification degree is defined similarly by using holomorphic jets $j_{h o l}^{k} u$ instead of $j^{k} u$.

For a map $u$ with ramification degree $k$, we call $\sigma^{k+1}(J,(j, u), z)$ the principal holomorphic jet of the map $u$ at $z$.

We next describe the holomorphic principal jet of a smooth map $u: \Sigma \rightarrow M$ relative to $(j, J)$ in complex coordinates. We refer to [7] for further details of some relevant exposition.

Let $z=s+i t$ be a complex coordinate of $(\Sigma, j)$ centered at $z_{0}$, and choose real coordinates $\left(x_{1}, \ldots, x_{n}, y_{1}, \ldots, y_{n}\right)$ of $M$ centered at $p_{0}=u\left(z_{0}\right)$ for $j=1, \ldots, n$ such that

$$
\left.J \frac{\partial}{\partial x_{j}}\right|_{p_{0}}=\left.\frac{\partial}{\partial y_{j}}\right|_{p_{0}},\left.\quad J \frac{\partial}{\partial y_{j}}\right|_{p_{0}}=-\left.\frac{\partial}{\partial x_{j}}\right|_{p_{0}} .
$$

We denote the associated complex coordinates by $\left(w_{1}, \ldots, w_{n}\right)$ with $w_{j}=$ $x_{j}+\sqrt{-1} y_{j}$. If $j^{k} u\left(z_{0}\right)=0$, then we can expand the map $u$ into the Taylor polynomial

$$
u(z)=\sum_{\ell=0}^{k+1} \vec{a}_{\ell} z^{\ell} \bar{z}^{k-\ell}+o\left(|z|^{k+1}\right) .
$$

Combining $(2.2)$ and $j^{k} u(z)=0$, we derive:

Lemma 2.3. Suppose $u$ is $(j, J)$-holomorphic, i.e., $\bar{\partial}_{(j, J)} u=0$ and $j^{k} u(z)=0$. Then we have

$$
u(z)=\vec{a}_{k+1} z^{k+1}+o\left(|z|^{k+1}\right)
$$

with $a_{k+1} \in T_{p_{0}} M \cong H_{z_{0}}^{(k ; 0)}$. In particular, the principal jet of $(j, J)$-holomorphic map $u$ is holomorphic at any point $z$, and the ramification degree of $u$ is the same as the holomorphic ramification degree at any given point $z \in \Sigma$.

It is easy to check that the principal term $\vec{a}_{k+1} z^{k+1}$, regarded as an element in $\operatorname{Sym}_{\left(j_{z_{0}}, J_{u\left(z_{0}\right)}\right)}^{k+1}\left(T_{z_{0}} \Sigma, T_{u\left(z_{0}\right)} M\right)$, has the from

$$
j^{k+1} u\left(z_{0}\right)=d^{k+1} u\left(z_{0}\right)=\vec{a} \cdot d z^{\otimes(k+1)}, \quad \vec{a} \in T_{u\left(z_{0}\right)} M
$$

for any map $(j, J)$-holomorphic map $u$ with $j^{k} u\left(z_{0}\right)=0$.

Now, we immediately obtain the following characterization for a $(j, J)$ holomorphic map $u$ with $j^{k} u\left(z_{0}\right)=0$ to satisfy $j^{k+1} u\left(z_{0}\right)=0$.

Lemma 2.4. Let $u$ be a $(j, J)$-holomorphic map with $j^{k} u\left(z_{0}\right)=0$. Then $j^{k+1} u\left(z_{0}\right)=0$ if and only if $\sigma^{k+1}\left(J,(j, u), z_{0}\right)=0$. 
We note that when $k=1, \sigma(J,(j, u), z)=\partial_{(j, J)} u(z)$, the holomorphic part of the derivative $d u\left(z_{0}\right)$. This lemma is the higher jet analog to Lemma 2.2 [9] which will be the basis of our Fredholm setting for the evaluation transversality in higher jets.

\section{Fredholm framework}

Let $\Sigma$ be a compact orientable surface without boundary. We consider a triple $(J,(j, u), z)$ of compatible $J$ on $M, j$ complex structure on $\Sigma, u: \Sigma \rightarrow M$ a smooth map and a point $z \in \Sigma$.

Denote

$$
\begin{aligned}
\mathcal{F}(\Sigma, M ; \beta) & =\{(j, u) \mid j \in \mathcal{M}(\Sigma), u: \Sigma \rightarrow M,[u]=\beta\}, \\
\mathcal{F}_{1}(\Sigma, M ; \beta) & =\{((j, u), z) \mid(j, u) \in \mathcal{F}(\Sigma, M ; \beta), z \in \Sigma\}
\end{aligned}
$$

and consider the evaluation map

$$
\sigma^{k}: \mathcal{F}_{1}(\Sigma, M ; \beta) \rightarrow H^{(k, 0)} ;(J,(j, u), z) \mapsto \sigma^{k}(J,(j, u), z) .
$$

We will interpret this map as a section of some vector bundle over $\mathcal{J}_{\omega} \times$ $\mathcal{F}_{1}(\Sigma, M ; \beta)$.

For any given $(j, J)$ and $(u, z) \in \mathcal{F}_{1}(M ; \beta ; k)$, consider the vector space

$$
H_{(J,(j, u), z)}^{(k, 0)}:=\operatorname{Sym}_{j_{z}, J_{u(z)}}^{(k, 0)}\left(T_{z} \Sigma, T_{x} M\right)
$$

of dimension $2 n=\operatorname{dim} M$ and the vector bundle

$$
H_{1}^{(k, 0)}:=\bigcup_{(J,(j, u), z)} H_{(J,(j, u), z)}^{(k, 0)}
$$

over $\mathcal{J}_{\omega} \times \mathcal{F}_{1}(M ; \beta ; k)$ with $H_{(J,(j, u), z)}^{(k ; 0)}$ as its fiber, where the union is taken for all $(J,(j, u), z)$.

Then the following lemma is immediate by definition.

Lemma 3.1. The map

$$
\sigma^{k}: \mathcal{J}_{\omega} \times \mathcal{F}_{1}(M ; \beta ; k) \rightarrow H_{1}^{(k, 0)}(\Sigma \times M)
$$

defined by $\sigma(J,(j, u), z)=\pi_{(j, J)}^{\text {hol }}\left(d^{k} u(z)\right)$ is a smooth section of the vector bundle

$$
H_{1}^{(k, 0)}=H_{1}^{(k, 0)}(\Sigma \times M) \rightarrow \mathcal{J}_{\omega} \times \mathcal{F}_{1}(M ; \beta) .
$$

Remark 3.2. It is crucial in the Fredholm analysis that the section $\sigma^{k}$ can be defined on the space of smooth maps, not just on the moduli space of $J$ holomorphic maps.

We introduce the standard bundle

$$
\mathcal{H}^{\prime \prime}=\bigcup_{((j, u), J)} \mathcal{H}_{((j, u), J)}^{\prime \prime}, \quad \mathcal{H}_{((j, u), J)}^{\prime \prime}=\Omega_{j, J}^{(0,1)}\left(u^{*} T M\right)
$$


and define a map $\Upsilon_{k}$ by

$$
\Upsilon_{k}(J,(j, u), z)=\left(\bar{\partial}(J,(j, u)) ; \sigma^{k}(J,(j, u), z)\right),
$$

where we denote

$$
\bar{\partial}(J,(j, u)):=\bar{\partial}_{j, J}(u)=(d u)_{j, J}^{(0,1)}=\frac{d u+J d u j}{2} .
$$

We denote by

$$
\pi_{1}: \mathcal{J}_{\omega} \times \mathcal{F}_{1}(M ; \beta) \rightarrow \mathcal{J}_{\omega} \times \mathcal{F}(M ; \beta)
$$

the forgetful map of the marked point and consider the fiber product

$$
\mathcal{H}^{\prime \prime} \times_{\pi_{1}} H_{1}^{(k, 0)}
$$

of the two bundles,

$$
\mathcal{H}^{\prime \prime} \rightarrow \mathcal{J}_{\omega} \times \mathcal{F}(M ; \beta)
$$

and

$$
H_{1}^{(k, 0)} \rightarrow \mathcal{J}_{\omega} \times \mathcal{F}_{1}(M ; \beta) \rightarrow \mathcal{J}_{\omega} \times \mathcal{F}(\Sigma, M ; \beta) .
$$

More explicitly, we have

$$
\begin{aligned}
& \mathcal{H}^{\prime \prime} \times_{\pi_{1}} H_{1}^{(k, 0)} \\
:= & \left\{\left(\eta, \zeta_{0} ; J,(j, u), z\right) \mid \eta \in \mathcal{H}_{(J,(j, u))}^{\prime \prime}, \zeta_{0} \in H_{(J,(j, u), z)}^{(k, 0)},(J,(j, u), z) \in \mathcal{F}_{1}(M ; \beta)\right\} .
\end{aligned}
$$

We regard the fiber product as a bundle over $\mathcal{J}_{\omega} \times \mathcal{F}_{1}(M ; \beta)$ whose fiber at $(J,(j, u), z)$ is given by

$$
\mathcal{H}_{(J,(j, u))}^{\prime \prime} \oplus H_{(J,(j, u), z)}^{(k, 0)} .
$$

Then $\Upsilon_{k}$ defines a smooth map

$$
\Upsilon_{k}: \mathcal{J}_{\omega} \times \mathcal{F}_{1}(M ; \beta) \rightarrow \mathcal{H}^{\prime \prime} \times_{\pi_{1}} H_{1}^{(k, 0)}
$$

which becomes a smooth section of this vector bundle.

One can generalize the above discussion by considering arbitrary finite number of marked points and holomorphic $n$-jets, not just $\sigma^{n}$.

Let $\Sigma$ be a closed Riemann surface. We denote by $\widetilde{\operatorname{Conf}}_{k}(\Sigma) \subset \Sigma^{k}$ the set of $k$ ordered distinct points on $\Sigma$, and

$$
\widetilde{\operatorname{Conf}}(\Sigma)=\bigcup_{k=0}^{\infty} \widetilde{\operatorname{Conf}}_{k}(\Sigma) \text {. }
$$

For each given $k$ distinct points $\vec{z}=\left\{z_{1}, \ldots, z_{k}\right\}$, we consider the decoration of integers $n_{i}$ assigned at $z_{i}$ 's. We call $k$ the length of the configuration $\vec{z} \in$ $\widetilde{\operatorname{Conf}}(\Sigma)$. We denote $\vec{n}=\left\{n_{1}, \ldots, n_{k}\right\}$ and $K=\{1, \ldots, k\}$. For given $k \leq k^{\prime}$ with $K^{\prime}=\left\{1, \ldots, k^{\prime}\right\}$, we decompose

$$
K^{\prime}=K \cup\left(K^{\prime} \backslash K\right) \text {. }
$$

Definition 3.3. Consider the set of pairs $(k ; \vec{n})$ with $\vec{n} \in \mathbb{Z}^{k}$, where $k=$ $\operatorname{leng}(\vec{n})$. We say $\left(k^{\prime} ; \vec{n}^{\prime}\right)<(k ; \vec{n})$ if

$$
k \leq k^{\prime} \text {, and } n_{i} \leq n_{i}^{\prime} \text { for all } i \in K \subset K^{\prime} .
$$


Remark 3.4. This definition of partial order is consistent with the lower semicontinuity of the ramification degree under the limit of a sequence of $J$-holomorphic maps in $C^{\infty}$-topology.

We denote

$$
\mathcal{F}_{k}(M ; \beta)=\left\{((j, u), \vec{z}) \mid[u]=\beta, \vec{z}=\left(z_{1}, \ldots, z_{k}\right)\right\}
$$

and define

$\widetilde{\mathcal{M}}_{g, k}^{=}(M ; \beta ; \vec{n})=\left\{((j, u), z) \in \mathcal{F}_{k}(M ; \beta) \mid \bar{\partial}_{(j, J)} u=0, \operatorname{deg}_{z_{i}}=n_{i}, \operatorname{Crit}(u) \supset \vec{z}\right\}$.

Then since the cardinality of and the degrees of ramification points of a pseudoholomorphic map are finite (see [6], [10] for the proof), we immediately have:

Lemma 3.5. Let $J$ be any almost complex structure. Denote by $\widetilde{\mathcal{M}}_{g}^{(k ; \vec{n})}(M, J ; \beta)$ the image of the forgetful map

$$
\mathfrak{f o r g e t}_{(k ; \vec{n})}: \widetilde{\mathcal{M}}_{g, k}^{=}(M, J ; \beta ; \vec{n}) \rightarrow \widetilde{\mathcal{M}}_{g}(M, J ; \beta) .
$$

Then we have the decomposition

$$
\widetilde{\mathcal{M}}_{g}(M, J ; \beta)=\bigcup_{(k ; \vec{n})} \widetilde{\mathcal{M}}_{g}^{(k ; \vec{n})}(M, J ; \beta) .
$$

We call the $\widetilde{\mathcal{M}}_{g}^{(k ; \vec{n})}(M, J ; \beta)$ the $(k ; \vec{n})$-stratum of $\widetilde{\mathcal{M}}_{g}(M, J ; \beta)$. For general $J$, the union in (3.3) may not be a finite union and the strata in $\widetilde{\mathcal{M}}_{g}(M, J ; \beta)$ may not be smooth.

We also define the union

$$
\widetilde{\mathcal{M}}_{g, \leq}^{(k ; \vec{n})}(M, J ; \beta)=\bigcup_{\left(k^{\prime} ; \vec{n}^{\prime}\right) \leq(k ; \vec{n})} \widetilde{\mathcal{M}}_{g}^{\left(k^{\prime} ; \vec{n}^{\prime}\right)}(M, J ; \beta)
$$

which is the closure of $\widetilde{\mathcal{M}}_{g}^{(k ; \vec{n})}(M, J ; \beta)$ in $\widetilde{\mathcal{M}}_{g}(M, J ; \beta)$ in $C^{\infty}$ topology.

The main purpose of the present paper is to analyze the structure of this decomposition and to establish certain stratawise transversality for a generic choice of $J$.

\section{Higher jet evaluation transversality}

In this section, we first formulate the precise version of stratawise transversality of higher jet evaluation maps. Then we will prove the transversality imitating the proof of the 1-jet evaluation transversality Zhu and the present author gave in [9].

\subsection{Statement}

Denote by $\pi_{k}: \mathcal{J}_{\omega} \times \mathcal{F}_{k}(M ; \beta) \rightarrow \mathcal{J}_{\omega} \times \mathcal{F}(M ; \beta)$ the forgetful map and consider the fiber product $\mathcal{H}^{\prime \prime} \times_{\pi_{k}} \prod_{i=1}^{k} J_{h o l}^{n_{i}}$. For each given $(k ; \vec{n})$, we consider a section

$$
\Upsilon_{k}^{\vec{n}}=\mathcal{J}_{\omega} \times \mathcal{F}_{k}(M ; \beta) \rightarrow \mathcal{H}^{\prime \prime} \times_{\pi_{k}} \prod_{i=1}^{k} J_{\text {hol }}^{n_{i}}
$$


given by

$$
\Upsilon_{k}^{\vec{n}}(J,(j, u), \vec{z})=\left(\bar{\partial}(J,(j, u)) ;\left(j_{\text {hol }}^{n_{1}}\left(z_{1}\right), \ldots, j_{\text {hol }}^{n_{k}}\left(z_{k}\right)\right)\right)
$$

Denote by $o_{E}$ to be the zero section of any vector bundle $E$. The following lemma immediately follows from the definition of $\Upsilon_{k}^{\vec{n}}$.

Lemma 4.1. For given $(k ; \vec{n})$, we have

$$
\widetilde{\mathcal{M}}_{g, k}(M ; \beta ; \vec{n})=\left(\Upsilon_{k}^{\vec{n}}\right)^{-1}\left(o_{\mathcal{H}^{\prime \prime} \times_{\pi_{k}} \prod_{i=1}^{k} J^{\left(n_{i}, 0\right)}}\right)
$$

and

$$
\widetilde{\mathcal{M}}_{g, \leq}^{(k ; \vec{n})}(M ; \beta ; k)=\mathfrak{f o r g e t}_{(k ; \vec{n})}\left(\widetilde{\mathcal{M}}_{g, k}(M ; \beta ; \vec{n})\right)
$$

This leads us to study the transversality property of $\Upsilon_{k}^{\vec{n}}$ with respect to the zero section $o_{\mathcal{H}^{\prime \prime} \times_{\pi_{k}} \prod_{i=1}^{k} J^{\left(n_{i}, 0\right)}} \subset \mathcal{H}^{\prime \prime} \times_{\pi_{k}} \prod_{i=1}^{k} J^{\left(n_{i}, 0\right)}$.

The following is the main theorem we prove in this section.

Theorem 4.2. There exists a subset $\mathcal{J}_{\omega}^{\text {ram }} \subset \mathcal{J}_{\omega}$ such that for any $J \in \mathcal{J}_{\omega}^{\text {ram }}$ and $\beta \in H_{2}(M)$ and $g \in \mathbb{N}$, the linearization

$$
D_{(J,(j, u), \vec{z})} \Upsilon_{k}^{\vec{n}}: T_{J} \mathcal{J}_{\omega} \times T_{((j, u), \vec{z})} \mathcal{F}_{k}(M, \beta) \rightarrow \mathcal{H}_{(J,(j, u))}^{\prime \prime} \oplus \bigoplus_{i=1}^{k} J_{h o l,(J,(j, u), \vec{z})}^{n_{i}}
$$

is surjective at all $(J,(j, u), \vec{z}) \in\left(\Upsilon_{k}^{\vec{n}}\right)^{-1}\left(o_{\mathcal{H}^{\prime \prime} \times_{\pi_{k}} \prod_{i=1}^{k} J_{h o l}^{n_{i}}}\right)$. In particular the set

$$
\left(\Upsilon_{k}^{\vec{n}}\right)^{-1}\left(o_{\mathcal{H}^{\prime \prime} \times_{\pi_{k}} \prod_{i=1}^{k} J_{h o l}^{n_{i}}}\right) \subset \mathcal{J}_{\omega} \times \mathcal{F}_{k}(M, \beta)
$$

is a submanifold of $\widetilde{\mathcal{M}}_{g, k}(M ; \beta)$ of codimension $\sum_{i=1}^{k} 2 n n_{i}$.

\subsection{Proof}

In this subsection, we give the proof of Theorem 4.2. The scheme of the proof is a generalization of the one used for the 1-jet transversality in [9] to the higher jets, which however requires more sophisticated choice of function spaces in the proof.

Proof of Theorem 4.2. We consider the smooth section

$$
\Upsilon_{k}^{\vec{n}}: \mathcal{J}_{\omega} \times \mathcal{F}_{g, k}(M, \beta) \rightarrow \mathcal{H}^{\prime \prime} \times_{\pi_{k}} \prod_{i=1}^{k} J^{\left(n_{i}, 0\right)}
$$

defined by

$$
\Upsilon_{k}^{\vec{n}}(J,(j, u), z)=\left(\bar{\partial}(J,(j, u)),\left(j_{\text {hol }}^{n_{1}}\left(J,(j, u), z_{1}\right), \ldots, j_{\text {hol }}^{n_{k}}\left(J,(j, u), z_{k}\right)\right)\right),
$$

where $z=\left\{z_{1}, \ldots, z_{k}\right\} \in \widetilde{\operatorname{Conf}}(\Sigma)$.

The linearization map of $j_{h o l}^{n_{i}}$ is the direct sum

$$
D_{(J,(j, u), z)} j_{h o l}^{n_{i}}=\bigoplus_{\ell=1}^{n_{i}} D_{\left(J,(j, u), z_{i}\right)} \sigma_{\ell}^{\vec{n} ; i}
$$


where $\sigma_{\ell}^{\vec{n} ; i}: \mathcal{F}_{k}(M, \beta) \rightarrow H_{\left(J,(j, u), z_{i}\right)}^{(\ell, 0)}$ is the evaluation of the $\ell$-th holomorphic derivative at $z_{i}$ for $\ell \leq n_{i}$. And the map

$$
D_{(J,(j, u), z)} \sigma_{\ell}^{n_{i}}: T_{J} \mathcal{J}_{\omega} \times T_{((j, u), \vec{z})} \mathcal{F}_{k}(M, \beta) \rightarrow H_{\left(J,(j, u), z_{i}\right)}^{(k ; 0)}
$$

is given by

$$
D_{(J,(j, u), z)} \sigma_{\ell}^{n_{i}}(B,(b, \xi), \vec{v})=\left(D_{J,(j, u)} \bar{\partial}(B,(b, \xi)), D_{(J,(j, u), \vec{z})}\left(\sigma_{\ell}^{\vec{n} ; i}\right)\left(B,(b, \xi), v_{i}\right)\right) .
$$

Here we have

$$
\left.D_{(J,(j, u), \vec{z})}\left(\sigma_{\ell}^{\vec{n} ; i}\right)(B,(b, \xi), \vec{v})\right)=D_{J,(j, u)} \sigma_{\ell}^{\vec{n} ; i}(B,(b, \xi))(\vec{z})+\nabla_{v_{i}}\left(\sigma^{\ell}(u)\right)\left(z_{i}\right)
$$

for $B \in T_{J} \mathcal{J}_{\omega}, b \in T_{j} \mathcal{M}(\Sigma), v_{i} \in T_{z_{i}} \Sigma$ and $\xi \in T_{u} \mathcal{F}(M ; \beta)$.

Some remarks concerning the necessary Banach manifold set-up of the map $\Upsilon$ are now in order:

(1) To make evaluating $j^{k} u$ at a point $z \in \Sigma$ make sense, we need to take at least $W^{k+1, p}$-completion with $p>2$ of $\mathcal{F}_{1}(\beta ; k)$ at $z$ so that $j^{k}(u)$ lies in $W^{1, p}$ at $z$ which is then continuous at $z$. We actually need to take $W^{N, p}$-completion of $\mathcal{F}_{1}(\beta ; k)$ with $N=N(\beta, k)$ sufficiently large so that the section $\Upsilon$ is differentiable and that Sard-Smale theorem can be applied.

(2) We provide $\mathcal{H}^{\prime \prime}$ with the topology of a $W^{N, p}$ Banach bundle.

(3) We also need to provide some Banach manifold structure on $\mathcal{J}_{\omega}$. We can borrow Floer's scheme [2] for this whose details we refer readers thereto.

We now complete the tangent space $T_{((j, u), z)} \mathcal{F}_{k}(M, \beta)$ by the $W^{N, p}$-norm with $N$ sufficiently large so that $N$ is at least

$$
N \geq \max \left\{3, \max _{i}\left\{n_{i}+2 \mid i=1, \ldots, k\right\}\right\} .
$$

The choice of $N$ will vary depending only on the homology class $\beta$ and the genus $g$. Recall that if $u$ is in $W^{N, p}, D_{J,(j, u)} \sigma^{\vec{n} ; i}(B,(b, \xi))$ and $\nabla_{v_{i}}(\sigma(u))$ are in $W^{N-n_{i}-1, p}$ with $N-n_{i}-1 \geq 1$. Therefore their evaluations at $z_{i}$ are well defined since any $W^{1, p}$-map is continuous.

At fixed $(J,(u, j), \vec{z})$ where we do linearization of $\Upsilon_{k}^{\vec{n}}$, we will write

$$
\begin{aligned}
\Omega_{N, p}^{0}\left(u^{*} T M\right) & :=W^{N, p}\left(u^{*} T M\right)=T_{u} \mathcal{F}^{N, p}(\Sigma, M ; \beta), \\
\Omega_{N-1, p}^{(0,1)}\left(u^{*} T M\right) & :=W^{N-1, p}\left(\Lambda_{(j, J)}^{(0,1)}\left(u^{*} T M\right)\right)
\end{aligned}
$$

for the simplicity of notations.

To prove Theorem 4.2 , we need to verify that at each given point $(J,(j, u), \vec{z})$ $\in\left(\Upsilon_{k}^{\vec{n}}\right)^{-1}\left(o_{\mathcal{H}^{\prime \prime} \times_{\pi_{k}} \prod_{i=1}^{k} J_{h o l}^{n_{i}}}\right)$, the system of equations

$$
\begin{aligned}
D_{J,(j, u)} \bar{\partial}(B,(b, \xi)) & =\gamma, \\
D_{J,(j, u)}\left(\sigma^{\vec{n} ; i}\right)(B,(b, \xi))\left(z_{i}\right)+\nabla_{v_{i}}\left(\sigma_{\ell}^{\vec{n} ; i}(u)\right) & =\zeta_{i ; \ell} \\
\text { for } \ell=1, \ldots, n_{i}, i=1, \ldots, k &
\end{aligned}
$$


has a solution $(B,(b, \xi), \vec{v})$ for each given data

$$
\gamma \in \Omega_{N-1, p}^{(0,1)}\left(u^{*} T M\right), \quad \zeta_{i ; \ell} \in H_{\left(J,(j, u), z_{i}\right)}^{(\ell, 0)}
$$

with $1 \leq \ell \leq n_{i}$ and $1 \leq i \leq k$. It will be enough to consider the triple with $b=0$ and $\vec{v}=0$ which we will assume from now on.

We now compute the linearization $\left.D_{(J,(j, u), z)} \sigma_{\ell}^{\vec{n} ; i}(B,(b, \xi), \vec{v})\right)$. First recall that $\sigma_{\ell}^{\vec{n} ; i}$ defines a section of the pull-back of the vector bundle $H_{1}^{(\ell, 0)} \rightarrow$ $\mathcal{F}_{1}(M ; \beta)$ to $\mathcal{F}_{k}(M ; \beta)$ via the forgetful map $\mathcal{F}_{k}(M ; \beta) \rightarrow \mathcal{F}_{1}(M ; \beta)$, and the linearization is meant to be the covariant linearization of the section. Note that computation of this linearization is local near $z_{i} \in \Sigma$, and so we can use coordinate calculations at $z_{i}$ and $u(z)$ as in Section 2. By $J$-complex linearity of $\nabla$ and the vanishing at $z_{i}$ of the $n_{i}$-jet $j^{n_{i}} u\left(z_{i}\right)$, it is easy to see that we have

$$
\left.D_{(J,(j, u), \vec{z})}\left(\sigma_{\ell}^{\vec{n} ; i}\right)(0,(0, \xi), 0)\right)(\vec{z})=\pi_{h o l}\left(\left(\nabla_{d u}\right)^{n_{i}} \xi\left(z_{i}\right)\right)
$$

as long as $1 \leq \ell \leq n_{i}$.

Remark 4.3. We would like to point out that for a general map $u$ the formula for $\left.D_{(J,(j, u), \vec{z})}\left(\sigma_{\ell}^{\vec{n} ; i}\right)(0,(0, \xi), 0)\right)(\vec{z})$ involve products of $\nabla_{d u}^{k} \xi$ and $\nabla^{j} u$ with $0 \leq$ $k, j \leq n_{i}$ and $k+j=n_{i}$ in addition to $\pi_{h o l}\left(\left(\nabla_{d u}\right)^{\ell} \xi\left(z_{i}\right)\right)$. Those terms with $1 \leq j \leq n_{i}$ will vanish by the condition $j^{n_{i}} u=0$.

Since $u$ is $(j, J)$-holomorphic, it also follows that

$$
\pi_{h o l}\left(\left(\nabla_{d u}\right)^{\ell} \xi\left(z_{i}\right)\right)=\left(\nabla_{d u}^{\prime}\right)^{\ell} \xi\left(z_{i}\right),
$$

where $\nabla_{d u}^{\prime}=\pi_{h o l} \nabla_{d u}$

Now we study solvability of (4.7)-(4.8) by applying the Fredholm alternative. We regard

$$
\Omega_{N-1, p}^{(0,1)}\left(u^{*} T M\right) \times \prod_{i=1}^{k} J_{h o l ;\left(J,(j, u), z_{i}\right)}^{n_{i}}
$$

as a Banach space with the norm

$$
\|\cdot\|_{N-1, p}+\sum_{i=1}^{k} \sum_{\ell=1}^{n_{i}}|\cdot|_{z_{i} ; \ell}
$$

where $|\cdot| z_{i} ; \ell$ any norm induced by an inner product on the $2 n$-dimensional vector space $H_{\left(J,(j, u), z_{i}\right)}^{(\ell, 0)} \cong \mathbb{C}^{n}$.

For the clarification of notations, we denote any natural pairing

$$
\Omega_{N-1, p}^{(0,1)} \times\left(\Omega_{N-1, p}^{(0,1)}\right)^{*} \rightarrow \mathbb{R}
$$

by $\langle\cdot, \cdot\rangle$ and the inner product on $H_{\left(J,(j, u), z_{i}\right)}^{(\ell, 0)}$ by $(\cdot, \cdot)_{z_{i}}$.

Remark 4.4. We emphasize that for the map

$$
\vec{z} \mapsto D_{J,(j, u)} \sigma_{\ell}^{\vec{n} ; i}(B,(b, \xi))\left(z_{i}\right)
$$


to be defined as a continuous map to $H_{z_{i}}^{(\ell, 0)}$, the map $u$ must be at least $W^{N, p}$ for $N \geq \max \left\{n_{i}+1\right\}$ near each $z_{i}$. On the other hand, as it will be clear from the discussion in Section 5 we need to reduce the regularity of the completed Sobolev space from $W^{N, p}$ to $W^{n_{i}+1, p}$ locally near at each $z_{i}$ respectively.

Due to this remark and the fact that $n_{i}$ vary over $i$, we will first consider the problem on a space with regularity weaker than $W^{N, p}$ but stronger than $W^{n_{i}+1, p}$ locally near at $z_{i}$. In the end of the proof, we will establish solvability of (4.7)-(4.8) on $W^{N, p}$ by applying the elliptic regularity result of the equation (4.7).

To overcome the fact that $n_{i}$ vary over $i$, we fix a choice of cut-off functions $\chi=\sum_{i} \chi_{i}$ so that $\operatorname{supp} \chi_{i} \in D_{i}$ and $\chi_{i} \equiv 1$ on $V_{i} \subset D_{i}$, and $D_{i}$ are disjoint from one another. Define a norm

$$
\|\xi\|_{\vec{n}+\overrightarrow{1} ; \vec{z}}=\sum_{i=1}^{k}\left\|\chi_{i} \xi\right\|_{n_{i}+1, p}
$$

and the space

$$
\Omega_{\vec{n}+\overrightarrow{1} ; \vec{z}}^{0}\left(u^{*} T M\right)=\left\{\xi \in W^{2, p} \mid\|\xi\|_{\vec{n}+\overrightarrow{1} ; \vec{z}}<\infty\right\}
$$

as the completion of $\Omega^{(0,1)}\left(u^{*} T M\right)$ with respect to the norm $\|\cdot\|_{\vec{n}+\overrightarrow{1} ; \vec{z}}$.

With this definition, we first consider the equations (4.7) and (4.8) for $\xi \in$ $\Omega_{\vec{n}+\overrightarrow{1} ; \vec{z}}^{0}\left(u^{*} T M\right)$ when $\gamma$ lies in $\Omega_{\vec{n} ; \vec{z}}^{(0,1)}\left(u^{*} T M\right)$. We will derive the solvability for the case $\Omega_{N-1, p}^{(0,1)}\left(u^{*} T M\right)$ afterwards by applying the elliptic regularity of the equation (4.7).

Proposition 4.5. The map (4.5)

$$
D \Upsilon_{k}^{\vec{n}}: T_{J} \mathcal{J}_{\omega} \times \Omega_{\vec{n}+\overrightarrow{1} ; \vec{z}}^{0}\left(u^{*} T M\right) \rightarrow \Omega_{\vec{n} ; \vec{z}}^{(0,1)}\left(u^{*} T M\right) \times \prod_{i} J_{h o l ; J,(j, u), z_{i}}^{n_{i}}=: \mathcal{B}
$$

restricted to the elements of the form $(B,(0, \xi), 0)$ is onto at any $(J,(u, j), \vec{z})$ that lies in $\left(\Upsilon_{k}^{\vec{n}}\right)^{-1}\left(o_{\mathcal{H}^{\prime \prime}} \times_{\pi_{k}} o_{\prod_{i}} J_{h o l}^{n_{i}}\right)=\widetilde{\mathcal{M}}_{g, k}(M, \beta ; \vec{n})$.

Proof. To prove the surjectivity, we will prove that the image of $D_{J,(j, u), \vec{z}} \Upsilon_{k}^{\vec{n}}$ is dense and closed in $\mathcal{B}$.

We start with the denseness. Let

$$
(\eta, \vec{\zeta}) \in\left(\Omega_{N-1, p}^{(0,1)}\left(u^{*} T M\right)\right)^{*} \times \prod_{i=1}^{k} J_{h o l ;\left(J,(j, u), z_{i}\right)}^{n_{i}}
$$

for $\vec{\zeta}=\left(\zeta_{i ; \ell}\right)$ such that

$$
\left\langle D_{J,(j, u)} \bar{\partial}_{j, J}(B,(0, \xi)), \eta\right\rangle+\sum_{i=1}^{k} \sum_{\ell=1}^{n_{i}}\left(D_{J,(j, u)} \sigma_{\ell}^{\vec{n} ; i}(B,(0, \xi))\left(z_{i}\right), \zeta_{i ; \ell}\right)_{z_{i}}=0
$$


for all $\xi \in \Omega_{\vec{n}+\overrightarrow{1}, p}^{0}\left(u^{*} T M\right)$ and $B$. It will be enough to consider smooth $\xi$ 's in our consideration of (4.11) since $\Omega^{0}\left(u^{*} T M\right) \hookrightarrow \Omega_{\vec{n} ; \vec{z}}^{0}\left(u^{*} T M\right)$ is dense. Under this assumption, we would like to show that $\eta=0=\zeta_{i, \ell}$.

By the above discussion on $D_{J,(j, u)} \bar{\partial}(B,(0, \xi))$ and $D_{J,(j, u)} \sigma_{\ell}^{\vec{n} ; i}(B,(0, \xi))\left(z_{i}\right)$, (4.11) is equivalent to

$$
\left\langle D_{u} \bar{\partial}_{(j, J)} \xi+\frac{1}{2} B \circ d u \circ j, \eta\right\rangle+\sum_{i, \ell}\left\langle\left(\nabla_{d u}^{\prime}\right)^{\ell} \xi, \delta_{z_{i}} \zeta_{i, \ell}\right\rangle=0
$$

for all $B$ and $\xi$ of $C^{\infty}$, where $\delta_{z_{0}}$ is the Dirac-delta function.

Lemma 4.6. Suppose $j^{n_{i}} u\left(z_{i}\right)=0$. Then we have

$$
\left(\nabla_{d u}^{\prime}\right)^{\ell} \xi\left(z_{i}\right)=\partial^{\ell} \xi\left(z_{i}\right)
$$

for all $1 \leq \ell \leq n_{i}$ and $i=1, \ldots, k$, where $\partial$ is the Dolbeault differential with respect to $(j, J)$.

Taking $B=0$ in (4.11) and applying Lemma 4.6, we obtain

$$
\left\langle D_{u} \bar{\partial}_{(j, J)} \xi, \eta\right\rangle+\sum_{i, \ell}\left\langle\partial^{\ell} \xi, \delta_{z_{i}} \zeta_{i, \ell}\right\rangle=0 \quad \text { for all } C^{\infty} \text { section } \xi
$$

Therefore by definition of the distribution derivatives, $\eta$ satisfies

$$
\left(D_{u} \bar{\partial}_{(j, J)}\right)^{\dagger} \eta+\sum_{i, \ell}\left(\partial^{\dagger}\right)^{\ell}\left(\delta_{z_{i}} \zeta_{i ; \ell}\right)=0
$$

as a distribution, i.e.,

$$
\left(D_{u} \bar{\partial}_{(j, J)}\right)^{\dagger} \eta=-\sum_{i, \ell}\left(\partial^{\dagger}\right)^{\ell}\left(\delta_{z_{i}} \zeta_{i ; \ell}\right)
$$

where $\left(D_{u} \bar{\partial}_{j, J}\right)^{\dagger}$ is the formal adjoint of $D_{u} \bar{\partial}_{j, J}$ whose symbol is the same as $D_{u} \partial_{j, J}$ and so as a first order differential operator. We also recall that the adjoint $\partial^{\dagger}$ of $\partial$ is a first order elliptic operator which has the same principal symbol as $-\bar{\partial}$. Since $\operatorname{supp}\left(\partial^{\dagger}\right)^{\ell}\left(\delta_{z_{i}} \zeta_{i, \ell}\right) \subset\left\{z_{1}, \ldots, z_{k}\right\}$, we have $\left(D_{u} \bar{\partial}_{(j, J)}\right)^{\dagger} \eta=$ 0 on $\Sigma \backslash\left\{z_{1}, \ldots, z_{k}\right\}$ as a distribution. Then by the elliptic regularity (see Theorem 13.4.1 [4] for example), $\eta$ must be smooth on $\Sigma \backslash\left\{z_{1}, \ldots, z_{k}\right\}$.

On the other hand, by setting $\xi=0$ in (4.12), we get

$$
\langle B \circ d u \circ j, \eta\rangle=0
$$

for all $B \in T_{J} \mathcal{J}_{\omega}$. From this identity, standard argument from [2], [6] shows that $\eta=0$ in a small neighborhood of any somewhere injective point in $\Sigma \backslash$ $\left\{z_{1}, \ldots, z_{k}\right\}$. Such a somewhere injective point exists by the hypothesis of $u$ being somewhere injective (see Notation in the introduction) and the fact that the set of somewhere injective points is open and dense in the domain under the hypothesis (see [6]). Then by the unique continuation theorem, we conclude that $\eta=0$ on $\Sigma \backslash\left\{z_{1}, \ldots, z_{k}\right\}$ and so the support of $\eta$ as a distribution on $\Sigma$ is contained at the subset $\left\{z_{1}, \ldots, z_{k}\right\}$ of $\Sigma$.

We will postpone the proof of the following lemma till Section 5 . 
Lemma 4.7. $\eta$ is a distributional solution of $\left(D_{u} \bar{\partial}_{j, J}\right)^{\dagger} \eta=0$ on $\Sigma$ and so continuous. In particular, we have $\eta=0$ in $\left(\Omega_{(N-1, p)}^{(0,1)}\left(u^{*} T M\right)\right)^{*}$.

Once we know $\eta=0$, the equation (4.11) is reduced to

$$
\sum_{i=1}^{k} \sum_{\ell=1}^{n_{i}}\left(D_{J,(j, u)} \sigma(B,(0, \xi))\left(z_{i}\right), \zeta_{i ; \ell}\right)_{z_{i}}=0 .
$$

It remains to show that $\zeta_{i ; \ell}=0$. By considering $\xi$ supported in a disjoint union of small neighborhoods of $z_{i}$ 's, we obtain

$$
\sum_{\ell=1}^{n_{i}}\left(D_{J,(j, u)} \sigma(B,(0, \xi))\left(z_{i}\right), \zeta_{i ; \ell}\right)_{z_{i}}=0
$$

for all such $\xi$. Therefore to show $\zeta_{i ; \ell}=0$ for all $1 \leq \ell \leq n_{i}$ at each $i$, we have only to show that the image of the evaluation map

$$
\xi \mapsto \sum_{\ell=1}^{n_{i}} D_{J,(j, u)} \sigma_{\ell}^{\vec{n} ; i}(0,(0, \xi))\left(z_{i}\right)=\sum_{\ell=1}^{n_{i}} \partial^{\ell} \xi\left(z_{i}\right)
$$

is surjective onto $J_{h o l ; J,(j, u), z_{i}}^{n_{i}}$. First of all, we note that the $\ell$-th holomorphic jets of smooth $\xi$ for $\ell=1, \ldots, n_{i}$ are functionally independent and can be chosen freely separately. This reduces the surjectivity question order by order.

We focus on the $\ell$-th jet for each given $\ell=1, \ldots, n_{i}$. To show this surjectivity, we need to prove the existence of $\xi$ satisfying

$$
\partial^{\ell} \xi\left(z_{i}\right)=\zeta_{i ; \ell}
$$

at $z_{i}$ for any given $\zeta_{i ; \ell} \in H_{z_{i}}^{(\ell, 0}$. We can multiply a cut-off function $\chi$ to $\zeta_{i ; \ell}$ with $\chi \equiv 1$ to make $\zeta(z):=\chi(z) \zeta_{i ; \ell}$ and we may assume $\zeta$ is supported in a sufficient small neighborhood around $z_{i}$. If we write

$$
\sigma^{\ell}\left(J,(j, u), z_{i}\right)=\vec{a} \cdot d z^{\otimes \ell}
$$

with $\vec{a}(z)=\frac{\partial^{\ell} u}{\partial z^{\ell}}\left(z_{i}\right)$ and

$$
\zeta_{i ; \ell}\left(z_{i}\right)=b\left(z_{i}\right) \cdot d z^{\otimes \ell}
$$

(4.17) is reduced to the equation

$$
\frac{\partial^{\ell} \xi}{\partial z^{\ell}}\left(z_{i}\right)=b\left(z_{i}\right)
$$

By simply integrating this equation, we solve this equation in some neighborhood around $z_{0}$, which in turn solves $\partial^{\ell} \xi\left(z_{i}\right)=\zeta_{i, \ell}$. This finishes the proof of existence of a solution to (4.17) and hence to (4.8). This then proves that the image of (4.5) with $v=0$ is dense in

$$
\Omega_{\vec{n} ; \vec{z}}^{(0,1)}\left(u^{*} T M\right) \times \prod_{i}^{k} J_{h o l ;\left(J,(j, u), z_{i}\right)}^{n_{i}}
$$


as a map from $T_{J} \mathcal{J}_{\omega} \times \Omega_{\vec{n}+\overrightarrow{1} ; \vec{z}, p}^{0}\left(u^{*} T M\right)$. On the other hand by the elliptic regularity, it follows that for any fixed $J$, the image of $D_{J,(j, u)} \bar{\partial}$ from $T_{(u, j)} \mathcal{F}_{g, k}(\beta ; \vec{n})$ is closed in $\Omega_{\vec{n} ; \vec{z}}^{(0,1)}\left(u^{*} T M\right)$. We also note that $J_{h o l ; J,(j, u), z_{i}}^{\ell}$ is a finite dimensional vector space. These imply that the image of (4.5) is closed in (4.18). Therefore the map (4.17) is onto $\Omega_{\vec{n} ; \vec{z}}^{(0,1)}\left(u^{*} T M\right) \times \prod_{i=1}^{k} J_{h o l ; J,(j, u), z_{0}}^{n_{i}}$ as a map from $T_{J} \mathcal{J}_{\omega} \times \Omega_{\vec{n}+\overrightarrow{1} ; \vec{z}}^{0}\left(u^{*} T M\right)$. This finishes the proof of Proposition 4.5.

Now we finally go back to the study of (4.7)-(4.8) for the case of

$$
\gamma \in \Omega_{N-1, p}^{(0,1)}\left(u^{*} T M\right) \subset \Omega_{1, p}^{(0,1)}\left(u^{*} T M\right) .
$$

We recall $N \geq 3$. By the above analysis of the linearization $D \Upsilon_{k}^{\vec{n}}$ on $\Omega_{\vec{n} ; \vec{z}}^{(0,1)}$, we can find a solution $(B,(0, \xi), 0)$ of (4.7)-(4.8) with $B \in T_{J} \mathcal{J}_{\omega}$ and with $\xi$ as an element in $\Omega_{\vec{n}+\overrightarrow{1} ; \vec{z}}^{0}\left(u^{*} T M\right)$ for any $\vec{\zeta}=\left(\zeta_{i ; \ell}\right)$. Applying elliptic regularity to the equation (4.7), we derive that $\xi$ indeed lies in $W^{N, p}$ if $\gamma \in W^{N-1, p}$, and hence in $\Omega_{N, p}^{0}\left(u^{*} T M\right)$.

Therefore the map (4.5) is onto, i.e., $\Upsilon_{k}^{\vec{n}}$ is transverse to the submanifold

$$
o_{\mathcal{H}^{\prime \prime} \times \pi_{k}} \prod_{i=1}^{k} J_{h o l}^{n_{i}} \subset \mathcal{H}^{\prime \prime} \times_{\pi_{k}} \prod_{i=1}^{k} J_{h o l}^{n_{i}} .
$$

This finishes the proof of Theorem 4.2.

It follows from definition that

$$
\widetilde{\mathcal{M}}_{g, k}(M, \beta ; \vec{n})=\left(\Upsilon_{k}^{\vec{n}}\right)^{-1}\left(o_{\mathcal{H}^{\prime \prime} \times_{\pi_{k}} \prod_{i=1}^{k} J_{h o l}^{n_{i}}}\right)
$$

and we have the natural projection

$$
\pi: \widetilde{\mathcal{M}}_{g, k}(M, \beta ; \vec{n}) \rightarrow \mathcal{J}_{\omega}
$$

Then we have

$$
\widetilde{\mathcal{M}}_{g, k}(M, J ; \beta ; \vec{n})=\widetilde{\mathcal{M}}_{g, k}(M, \beta ; \vec{n}) \cap \pi^{-1}(J) .
$$

We denote

$$
\mathcal{J}_{\omega}^{g, k, \vec{n}}=\text { the set of regular values of } \pi \text {. }
$$

An immediate corollary of this proposition and the discussion in Section 3 is:

Corollary 4.8. For any $J \in \mathcal{J}_{\omega}^{g, k, \vec{n}}, \widetilde{\mathcal{M}}_{g, k}(M, J ; \beta ; \vec{n})$ is a smooth manifold of $\widetilde{\mathcal{M}}_{g, k}(M, J ; \beta)$ of codimension $\left(\sum_{i=1}^{k} 2 n n_{i}\right)$.

Proof. Here each $2 n n_{i}$ comes from the vanishing of $n_{i}$ derivatives at a marked point $z_{i}$ and -2 comes from the location of marked point $z_{i}$ in $\Sigma$. 
Now we set

$$
\mathcal{J}_{\omega}^{\text {ram }}=\bigcap_{g \in \mathbb{Z}_{\geq 0}} \bigcap_{k \in \mathbb{N}} \bigcap_{\vec{n} \in \mathbb{N}^{k}} \mathcal{J}_{\omega}^{g, k, \vec{n}} .
$$

Obviously $\mathcal{J}_{\omega}^{\text {ram }}$ is a subset of $\subset \mathcal{J}_{\omega}$ of second category.

\section{Removal of singularity: Proof of Lemma 4.7}

In this section, we prove Lemma 4.7. Our primary goal is to prove

$$
\left\langle D_{u} \bar{\partial}_{(j, J)} \xi, \eta\right\rangle=0
$$

for all smooth $\xi \in \Omega^{0}\left(u^{*} T M\right)$, i.e., $\eta$ is a distributional solution of $\left(D_{u} \bar{\partial}_{(j, J)}\right)^{\dagger} \eta$ $=0$ on the whole $\Sigma$, not just on $\Sigma \backslash\left\{z_{1}, \ldots, z_{k}\right\}$ which was shown in Section 4 . In addition, $\eta$ is a continuous linear functional on $\Omega_{N-1, p}^{(0,1)}\left(u^{*} T M\right)$.

We start with (4.14), which is

$$
\left\langle D_{u} \bar{\partial}_{(j, J)} \xi, \eta\right\rangle+\sum_{i, \ell}\left\langle\partial^{\ell} \xi, \delta_{z_{i}} \zeta_{i, \ell}\right\rangle=0
$$

for all $\xi$ of $C^{\infty}$. We first simplify the expression of the pairing $\left\langle D_{u} \bar{\partial}_{(j, J)} \xi, \eta\right\rangle$ knowing that $\operatorname{supp} \eta \subset\left\{z_{1}, \ldots, z_{k}\right\}$. Recall the well-known computation

$$
D_{J,(j, u)} \bar{\partial}(0,(0, \xi))=D_{u} \bar{\partial}_{j, J} \xi=\left(\nabla_{d u} \xi\right)_{(j, J)}^{(1,0)}+T_{(j, J)}^{(1,0)}(d u, \xi)
$$

with respect to a $J$-complex connection $\nabla$ and its torsion tensor $T$. Here we denote

$$
T_{(j, J)}^{(1,0)}(d u, \xi)=\frac{1}{2}(T(d u, \xi)+J T(d u \circ j, \xi)) .
$$

From this it follows that

$$
\nabla_{d u}^{\ell}\left(T_{(j, J)}^{(1,0)}(d u, \xi)\right)\left(z_{i}\right)=0
$$

for all $0 \leq \ell \leq n_{i}-1$, provided $j^{n_{i}} u\left(z_{i}\right)=0$.

Now we simplify the expression of $\left(\nabla_{d u} \xi\right)_{(j, J)}^{(0,1)}$ in complex coordinates $z$ at $z_{0}$. Let $x_{0}=u\left(z_{0}\right)$, and identify a neighborhood of $z_{0}$ with an open subset of $\mathbb{C}$ and a neighborhood of $x_{0}$ with an open set in $T_{x_{0}} M$. Then if we identify $\left(T_{x_{0}} M, J_{x_{0}}\right) \cong \mathbb{C}^{n}$, we can write the operator

$$
\left(\nabla_{d u} \xi\right)_{(j, J)}^{(0,1)}=\bar{\partial} \xi+C \cdot \partial \xi+D \cdot \xi
$$

where in a neighborhood of $z_{0}, \partial, \bar{\partial}$ are the standard Cauchy-Riemann operators on $\mathbb{C}^{n}$ and $C=C(x), D=D(x)$ are smooth pointwise (matrix) multiplication operators whose coefficients depend only on $M$ and $J$ and satisfies

$$
C\left(x_{0}\right)=0=D\left(x_{0}\right), \quad x_{0}=u\left(z_{0}\right) .
$$

Adding (5.3) and (5.5), we can write

$$
D_{J,(j, u)} \bar{\partial}(0,(0, \xi))=\bar{\partial} \xi+E \cdot \partial \xi+F \cdot \xi
$$

(See [10], [9]). The following is a simple consequence of the chain rule and (5.4) and (5.6). 
Lemma 5.1. Suppose $j^{n_{i}} u\left(z_{0}\right)=0$ and let $E=E(u(z)), F=F(u(z))$ in the above formula. Then we have

$$
\nabla_{d u}^{k}(E \cdot \xi)\left(z_{0}\right)=0=\nabla_{d u}^{k}(F \cdot \xi)\left(z_{0}\right)
$$

for all $0 \leq k \leq n_{i}$.

Let $z$ be a complex coordinate centered at $z_{0}$ and $\left(w_{1}, \ldots, w_{n}\right)$ be the complex coordinates on $M$ regarded as coordinates on a neighborhood of $u\left(z_{0}\right)$. We consider the standard metric

$$
h=\frac{\sqrt{-1}}{2} d z d \bar{z}
$$

on a neighborhood $U$ of $z_{0}$ and with respect to the coordinates $\left(w_{1}, \ldots, w_{n}\right)$ we fix any Hermitian metric on $\mathbb{C}^{n}$.

We fix complex coordinates satisfying (2.2) at each $z_{i}$ and denote by $\bar{\partial}$ the Dolbeault differential with respect to the complex coordinates on the corresponding coordinate neighborhoods respectively. We fix cut-off functions $\chi_{i}$ whose support supp $\chi_{i}$ is contained in $D_{i}$ a neighborhood of $z_{i}$ respectively. We also assume that $D_{i}$ 's are disjoint from one another.

By multiplying a cut-off function $\chi=\sum_{i} \chi_{i}$ to $\xi$, the map $\bar{\partial}_{\chi}$ defined by

$$
\bar{\partial}_{\chi}(\xi):=\sum_{i} \bar{\partial}\left(\chi_{i} \xi\right)
$$

gives rise to a well-defined continuous operator from $\Omega_{\vec{n}+\overrightarrow{1} ; \vec{z}}^{0}\left(u^{*} T M\right)$ to $\Omega_{\vec{n} ; \vec{z}}^{(0,1)}$.

The following proposition will be crucial in our proof. Here our choice of the above particular mixed Sobolev norm enters in the proof in a crucial way similar as in the proof of Lemma $2.5[9]$.

Proposition 5.2. Let $\eta \in\left(\Omega_{\vec{n} ; \vec{z}}^{(0,1)}\left(u^{*} T M\right)\right)^{*}$ be the distribution on $\Sigma$ obtained above. Then for any smooth section $\xi$ of $u^{*}(T M)$, we have

$$
\left\langle D_{u} \bar{\partial}_{(j, J)} \xi, \eta\right\rangle=\left\langle\bar{\partial}_{\chi} \xi, \eta\right\rangle
$$

Proof. We have already shown that $\eta$ is a distribution with $\operatorname{supp} \eta \subset\left\{z_{1}, \ldots, z_{k}\right\}$. By the structure theorem on the distributions supported at a point (see Section 4.5 , especially p. 119, of [5]), we have

$$
\eta=\sum_{i} P_{i}\left(\frac{\partial}{\partial s}, \frac{\partial}{\partial t}\right)\left(\delta_{z_{i}}\right),
$$

where $z=s+i t$ is the given complex coordinates at $z_{i}$ and $P_{i}\left(\frac{\partial}{\partial s}, \frac{\partial}{\partial t}\right)$ is a differential operator associated with the polynomial $P_{i}$ of two variables. Furthermore since $\eta \in\left(W^{n_{i}, p}\right)^{*}$, the degree of $P_{i}$ must be less than equal to $n_{i}-1$ : This is because the 'evaluation at a point of the $n_{i}$-th derivative of $W^{n_{i}, p}$ map does not define a continuous functional on $W^{n_{i}, p}$. 
By multiplying a cut-off function $\chi=\sum_{i} \chi_{i}$ and using the support condition on $\eta$, we have

$$
\left\langle D_{u} \bar{\partial}_{(j, J)} \xi, \eta\right\rangle=\sum_{i}\left\langle D_{u} \bar{\partial}_{(j, J)}\left(\chi_{i} \xi\right), \eta\right\rangle
$$

Therefore to prove the lemma, it is enough to prove

$$
\left\langle D_{u} \bar{\partial}_{(j, J)}\left(\chi_{i} \xi\right), \eta\right\rangle=\left\langle\bar{\partial}\left(\chi_{i} \xi\right), \eta\right\rangle
$$

for each $i$. We now recall

$$
D_{u} \bar{\partial}_{(j, J)} \xi=\bar{\partial} \xi+E \cdot \partial \xi+F \cdot \xi
$$

in coordinates at $z_{i}$, where $E$ and $F$ are zero-order matrix operators with $E\left(z_{i}\right)=0=F\left(z_{i}\right)$ satisfying (5.7). Therefore by (5.8), we derive

$$
\left\langle E \cdot \partial\left(\chi_{i} \xi\right)+F \cdot\left(\chi_{i} \xi\right), \eta\right\rangle=\left\langle E \cdot \partial\left(\chi_{i} \xi\right)+F \cdot\left(\chi_{i} \xi\right), P_{i}\left(\frac{\partial}{\partial s}, \frac{\partial}{\partial t}\right) \delta_{z_{i}}\right\rangle .
$$

By writing out

$$
P_{i}\left(\frac{\partial}{\partial s}, \frac{\partial}{\partial t}\right)=\sum_{0 \leq a+b \leq n_{i}-1} \vec{a}_{a, b} \frac{\partial^{a+b}}{\partial s^{a} \partial t^{b}},
$$

integrating by parts and then applying Lemma 5.1, we obtain

$$
\begin{aligned}
& \left\langle E \cdot \partial\left(\chi_{i} \xi\right)+F \cdot\left(\chi_{i} \xi\right), \eta\right\rangle \\
= & \sum_{0 \leq a+b \leq n_{i}-1}(-1)^{a+b}\left\langle\frac{\partial^{a+b}}{\partial s^{a} \partial t^{b}}\left(E \cdot \partial\left(\chi_{i} \xi\right)+F \cdot\left(\chi_{i} \xi\right)\right), \vec{a}_{a, b} \delta_{z_{i}}\right\rangle \\
= & \sum_{0 \leq a+b \leq n_{1}-1}(-1)^{a+b}\left(\frac{\partial^{a+b}}{\partial s^{a} \partial t^{b}}(E \cdot \partial(\chi \xi)+F \cdot(\chi \xi))\left(z_{0}\right), \vec{a}_{a, b}\right)_{z_{i}}=0 .
\end{aligned}
$$

Therefore we obtain

$$
\left\langle D_{u} \bar{\partial}_{(j, J)} \xi, \eta\right\rangle=\left\langle\bar{\partial}_{\chi} \xi+E \cdot \partial \xi+F \cdot \xi, \eta\right\rangle=\left\langle\bar{\partial}_{\chi} \xi, \eta\right\rangle
$$

which finishes the proof.

This lemma then implies that (5.2) is equivalent to

$$
\left\langle\bar{\partial}_{\chi} \xi, \eta\right\rangle+\sum_{i=1}^{k} \sum_{\ell=1}^{n_{i}}\left\langle\partial^{\ell} \xi, \delta_{z_{i}} \zeta_{i ; \ell}\right\rangle=0 \quad \text { for all } \xi
$$

Express $\partial^{\ell} \xi$ as

$$
\partial^{\ell} \xi\left(z_{i}\right)=a_{i ; \ell}(z) d z^{\otimes \ell}
$$

in $D_{i}$ in coordinates with $a_{i ; \ell}\left(z_{i}\right) \in \mathbb{C}^{n}$. We decompose $\xi$ as

$$
\xi=\widetilde{\xi}(z)+\frac{1}{\ell !} \sum_{i} \sum_{\ell} \chi_{i}(z)\left(z-z_{i}\right)^{\ell} a_{i ; \ell}\left(z_{i}\right)
$$


by defining $\widetilde{\xi}$ by

$$
\widetilde{\xi}(z)=\xi(z)-\frac{1}{\ell !} \sum_{i} \sum_{\ell} \chi_{i}(z)\left(z-z_{i}\right)^{\ell} a_{i ; \ell}\left(z_{i}\right) .
$$

Our choice of this decomposition is dictated by the fact

$$
\partial^{\ell}\left(\chi_{i}(z)\left(z-z_{i}\right)^{\ell} a_{i ; \ell}\left(z_{i}\right)\right)\left(z_{i}\right)=\ell ! \cdot a_{i ; \ell}\left(z_{i}\right) \cdot d z^{\otimes \ell} .
$$

Then $\widetilde{\xi}$ is a smooth section on $\Sigma$, and satisfies

$$
\partial^{\ell} \widetilde{\xi}\left(z_{i}\right)=0
$$

and

$$
\bar{\partial} \widetilde{\xi}=\bar{\partial} \xi \quad \text { on } V_{i}
$$

for all $i, \ell$. Therefore applying (5.9) to $\widetilde{\xi}$ instead of $\xi$, we obtain

$$
\left\langle\bar{\partial}_{\chi} \widetilde{\xi}, \eta\right\rangle+\sum_{i, \ell}\left\langle\partial^{\ell}\left(\chi_{i} \widetilde{\xi}\right), \delta_{z_{i}} \zeta_{i ; \ell}\right\rangle=0
$$

But we have

$$
\left\langle\bar{\partial}_{\chi} \widetilde{\xi}, \eta\right\rangle=\left\langle\bar{\partial}_{\chi} \xi, \eta\right\rangle
$$

since $\bar{\partial} \widetilde{\xi}=\bar{\partial} \xi$ on $V_{i}$ and $\operatorname{supp} \eta \subset\left\{z_{1}, \ldots, z_{k}\right\}$. Again using the support property $\operatorname{supp} \eta \subset\left\{z_{1}, \ldots, z_{k}\right\}$ and (5.10), (5.11), we derive

$$
\begin{aligned}
\left\langle\partial^{\ell} \widetilde{\xi}, \delta_{z_{i}} \zeta_{i, \ell}\right\rangle & =\left\langle\partial^{\ell} \xi, \delta_{z_{i}} \zeta_{i ; \ell}\right\rangle-\left\langle\partial^{\ell}\left(\chi(z)\left(z-z_{i}\right)^{\ell} a_{i ; \ell}\left(z_{i}\right)\right), \delta_{z_{i}} \zeta_{i ; \ell}\right\rangle \\
& =\left(\partial^{\ell} \xi, \zeta_{i ; \ell}\right)_{z_{i}}-\left(a_{i ; \ell}\left(z_{i}\right) d z^{\otimes \ell}, \zeta_{i ; \ell}\right)_{z_{i}} \\
& =\left(\partial^{\ell} \xi\left(z_{i}\right)-a_{i ; \ell}\left(z_{i}\right) d z^{\otimes \ell}, \zeta_{i ; \ell}\right)_{z_{i}}=0
\end{aligned}
$$

where the equality next to the last comes from (5.10). Substituting (5.12) and (5.13) into (5.9), we obtain $\left\langle\bar{\partial}_{\chi} \xi, \eta\right\rangle=0$ and hence (5.1) follows.

Since $(5.1)$ holds for all $\xi$, we have proved that $\eta$ is a distributional solution of $\left(D_{u} \bar{\partial}_{j, J}\right)^{\dagger} \eta=0$ on $\Sigma$. This finishes the proof of the first part of the lemma.

This then implies that $\eta$ extends continuously at $z_{i}$ if $\eta \in \Omega_{\vec{n} ; \vec{z}, p}^{(0,1)}\left(u^{*} T M\right)$. Hence we have proved $\eta \equiv 0$ since we already know that $\eta=0$ on $\Sigma \backslash$ $\left\{z_{0}, \ldots, z_{k}\right\}$.

\section{Stratawise transversality and finiteness of ramification profiles}

In this section, we apply the stratawise transversality result to prove a finiteness result on the types of singularities of $J$-holomorphic maps $u: \Sigma \rightarrow M$ with fixed homology class $f_{*}[\Sigma]=\beta \in H_{2}(M, \mathbb{Z})$. The case $n=1$ corresponds to the case where both domain and target are Riemann surfaces. In this case, finiteness of ramification profiles follows from the classical Hurewitz formula. Therefore we will assume $n \geq 2$ in this section.

Next for each given non-constant $J$-holomorphic map $(j, u)$, we associate to it the ramification profile: 
Definition 6.1. A ramification profile, denoted by $\operatorname{ram}(u)$, of $(j, u)$ is a pair $(N ; \lambda)$ with $N \in \mathbb{Z}_{\geq 0}$ and a partition $\lambda=\left(\lambda_{1}, \ldots, \lambda_{L}\right) \vdash N$ of $N$ such that

$$
\text { Crit } u=\left\{z_{1}, \ldots, z_{k}\right\} \subset \Sigma, \quad k=\sum_{\ell=1}^{L} a_{\ell},
$$

where $L=|\lambda|$ is the length of the partition and $\lambda=\left(d_{1}^{a_{1}} \cdots d_{L}^{a_{L}}\right)$ to denote the partition that has $a_{j}$ points of $z_{i}$ that has the same degree $d_{\ell}$. We call $N$ the total ramification degree of $(j, u)$.

We denote by $(k ; \vec{n})$ the pair $k \in \mathbb{Z}_{\geq 0}$ and $\vec{n} \in \mathbb{N}^{k}$ associated to the profile $(N ; \lambda)$ given as before in the introduction.

We have two kinds of immediate predecessors $\left(k^{\prime} ; \vec{n}^{\prime}\right)$ to $(k ; \vec{n})$.

(a) $\left(k ; \vec{n}^{\prime}\right)=\left(k ; \vec{n}+\vec{e}_{i}\right)$ for some $1 \leq i \leq k$ where we denote $\vec{n}+\vec{e}_{i}$ the decoration

$$
\left(n_{1}, \ldots, n_{i}+1, \ldots, n_{k}\right) .
$$

(b) $\left(k^{\prime} ; \vec{n}^{\prime}\right)=\left(k+1, \vec{n} \cup\left\{n_{k+1}\right\}\right)$ with $n_{k+1}=1$.

We have already proved that if $J \in \mathcal{J}_{\omega}^{\text {ram }}$, each moduli space $\mathcal{M}_{g, k}(M, J ; \beta, \vec{n})$ is a smooth manifold itself.

The following is an immediate consequence of Theorem 4.2, which relates two moduli spaces right next to each other in the partial order $<$.

Theorem 6.2. For $J \in \mathcal{J}_{\omega}^{\text {ram }}$ and $\beta \in H_{2}(M)$ and $g \in \mathbb{N}$, the following holds:

(1) For the type (a) of the immediate predecessor of $\left(k^{\prime} ; \vec{n}^{\prime}\right)=\left(k, \vec{n}+\vec{e}_{i}\right)$ for some $i=1, \ldots, k, \widetilde{\mathcal{M}}_{g, k}\left(J ; \beta, \vec{n}+\vec{e}_{i}\right)$ is a smooth submanifold of $\widetilde{\mathcal{M}}_{g, k}(J ; \beta, \vec{n})$ with its dimension $2 n$ smaller,

(2) For the type (b), the forgetful map

$$
\mathcal{M}_{g, k+1}\left(J ; \beta, \vec{n}+\vec{e}_{k+1}\right) \rightarrow \mathcal{M}_{g, k}(J ; \beta, \vec{n})
$$

is an embedding of codimension $2(n-1)$.

Proof. We start with the case (1). By definition, we have

$$
\widetilde{\mathcal{M}}_{g, k}\left(M, J ; \beta ; \vec{n}+\vec{e}_{i}\right) \subset \widetilde{\mathcal{M}}_{g, k}(M, J ; \beta ; \vec{n}) .
$$

Since we have chosen $J \in \mathcal{J}_{\omega}^{\text {ram }}$, Theorem 4.2 implies that both $\widetilde{\mathcal{M}}_{g, k}(M, J ; \beta ; \vec{n})$ and $\widetilde{\mathcal{M}}_{g, k}\left(M, J ; \beta ; \vec{n}+\vec{e}_{i}\right)$ are smooth manifolds and the latter has codimension $2 n$ in the former.

The case of immediate predecessor of the type (2) essentially follows from the proof of 1-jet evaluation transversality result of [9] (see Section 2 [9] more specifically). This finishes the proof.

Another immediate consequence of Theorem 4.2 is the following finiteness result. 
Theorem 6.3. Let $\beta \in H_{2}(M, \mathbb{Z})$ and $g$ be given. Then for any $J \in \mathcal{J}_{\omega}^{\text {ram }}$, the number of types of ramification profiles is not bigger than

$$
P\left(c_{1}(\beta)+(3-n)(g-1)\right),
$$

that is, the number of partitions of the integer $c_{1}(\beta)+(3-n)(g-1)$.

Proof. We have the natural projection

$$
\pi: \widetilde{\mathcal{M}}_{g, k}(M, \beta ; \vec{n}):=\bigcup_{J \in \mathcal{J}_{\omega}} \widetilde{\mathcal{M}}_{g, k}(M, J ; \beta ; \vec{n}) \rightarrow \mathcal{J}_{\omega} .
$$

The projection has index

$$
2\left(c_{1}(\beta)+n(1-g)\right)+2 k-\sum_{\ell=1}^{k} 2 n n_{\ell}=2\left(c_{1}(\beta)+n(1-g)\right)-\sum_{\ell=1}^{k} 2\left(n n_{\ell}-1\right)
$$

so for any regular value $J$, the moduli space

$$
\widetilde{\mathcal{M}}_{g, k}(M, J ; \beta ; \vec{n})=\Upsilon_{\ell}^{-1}\left(o_{\mathcal{H}^{\prime \prime}} \times o_{H^{\left(n_{\ell}, 0\right)}}\right) \cap \pi^{-1}(J)
$$

is of dimension

$$
2\left(c_{1}(\beta)+n(1-g)\right)-\sum_{\ell=1}^{k} 2\left(n n_{\ell}-1\right) .
$$

We consider the quotient

$$
\mathcal{M}_{g, k}(M, J ; \beta ; \vec{n}):=\widetilde{\mathcal{M}}_{g, k}(M, J ; \beta ; \vec{n}) / \operatorname{Aut}(\Sigma),
$$

where $\operatorname{Aut}(\Sigma)$ acts on marked Riemann surfaces $(\Sigma, j, z)$ by conformal equivalence then on the maps from them. For any $J \in \mathcal{J}_{\omega}^{\text {ram }}, \mathcal{M}_{g, k}(M, J ; \beta ; \vec{n})$ has its dimension

$$
2\left(c_{1}(\beta)+(3-n)(g-1)-\sum_{\ell=1}^{k}\left(n n_{\ell}-1\right)\right)
$$

as a smooth orbifold. Therefore, $\mathcal{M}_{g, k}(M, J ; \beta ; \vec{n})$ is empty whenever this dimension is negative. In other words, if $\mathcal{M}_{g, k}(M, J ; \beta ; \vec{n}) \neq \emptyset$, then we should have

$$
\sum_{\ell=1}^{k}\left(n n_{\ell}-1\right) \leq c_{1}(\beta)+(3-n)(g-1)
$$

In particular if $c_{1}(\beta)+(3-n)(g-1) \leq 0$, then for any element $(j, u) \in$ $\mathcal{M}_{g, k}(M, J ; \beta), u$ will be immersed.

On the other hand, we note that since we assume $n \geq 2, n n_{\ell}-1 \geq n-1>0$. Therefore if $c_{1}(\beta)+(3-n)(g-1)>0$, the inequality (6.2) implies that the number of admissible pairs $(k ; \vec{n})$ is not bigger than

$$
P\left(c_{1}(\beta)+(3-n)(g-1)\right),
$$

that is, the number of partitions of the integer $c_{1}(\beta)+(3-n)(g-1)$. This then finishes the proof of Theorem 6.3. 


\section{References}

[1] J.-F. Barrard, Courbes pseudo-holomorphes équisingulières en dimension 4, Bull. Soc. Math. France 128 (2000), no. 2, 179-206.

[2] A. Floer, The unregularized gradient flow of the symplectic action, Comm. Pure Appl. Math. 41 (1988), no. 6, 775-813.

[3] M. Hirsch, Differential Topology, Springer-Verlag, 1976.

[4] L. Hörmander, The Analysis of Linear Partial Differential Operators. II, SpringerVerlag, Berlin, 1983

[5] I. M. Gelfand and G. E. Shilov, Generalized Functions, vol 2, Academic Press, New York and London, 1968.

[6] D. McDuff, Examples of symplectic structures, Invent. Math. 89 (1987), no. 1, 13-36.

[7] _ Singularities of J-holomorphic curves in almost complex 4-manifolds, J. Geom. Anal. 2 (1992), no. 3, 249-266.

[8] Y.-G. Oh, Seidel's long exact sequence on Calabi-Yau manifolds, submitted, arXiv: 1002.1648.

[9] Y.-G. Oh and K. Zhu, Embedding property of J-holomorphic curves in Calabi-Yau manifolds for generic J, Asian J. Math. 13 (2009), no. 3, 323-340.

[10] J.-C. Sikorav, Some properties of holomorphic curves in almost complex manifolds, Holomorphic Curves in Symplectic Geometry, 165-189, Progr. Math., 117, Birkhauser, Basel, 1994.

Department of Mathematics

UNIVERSITY OF WISCONSIN

MADISON, WI, 53706

E-mail address: oh@math.wisc.edu 